\section{ENERGY-FILTERED IMAGING}

J. Bentley, Oak Ridge National Laboratory bentleyj@ornl.org

Over the several years that imaging energy filters have been available commercially, numerous and wide-ranging applications have demonstrated elemental mapping with a resolution approaching $1 \mathrm{~nm}$. A few reports have even shown resolutions $<0.4 \mathrm{~nm}$. Elemental mapping by energy-filtered transmission electron microscopy (EFTEM) is clearly an attractive and powerful tool, but some aspects of the techniques can be complex, with many pitfalls awaiting the unwary. This tutorial aims to cover some practical aspects of elemental mapping by EFTEM. It is based largely on the author's work at the ORNL SHaRE User Facility, where EFTEM research has been performed since 1994 with a Gatan imaging filter (GIF) interfaced to a Philips CM30T operated at $300 \mathrm{kV}$ with a $\mathrm{LaB}_{6}$ cathode. ${ }^{1-20}$ Most of the applications have been to metals and ceramics, emphasizing interfacial segregation and precipitation.

For quantitative composition mapping by EFTEM a number of interrelated parameters [field of view, resolution $(\delta)$, collection angle $(\beta)$, energy window or slit width $(\Delta)$, incident beam divergence $(\alpha)$, illumination uniformity, probe current $(i)$, drift rate, exposure time $(T)$, and local specimen thickness $(t)]$ should be properly selected and controlled. For example, the collection angle should be large enough for a strong signal, but not so large that the signal/background (S/B) and chromaticaberration-limited spatial resolution are both degraded. ${ }^{15}$ For optimum signal/noise with minimum exposure and justsufficient over-sampling, microscope magnification and on-chip

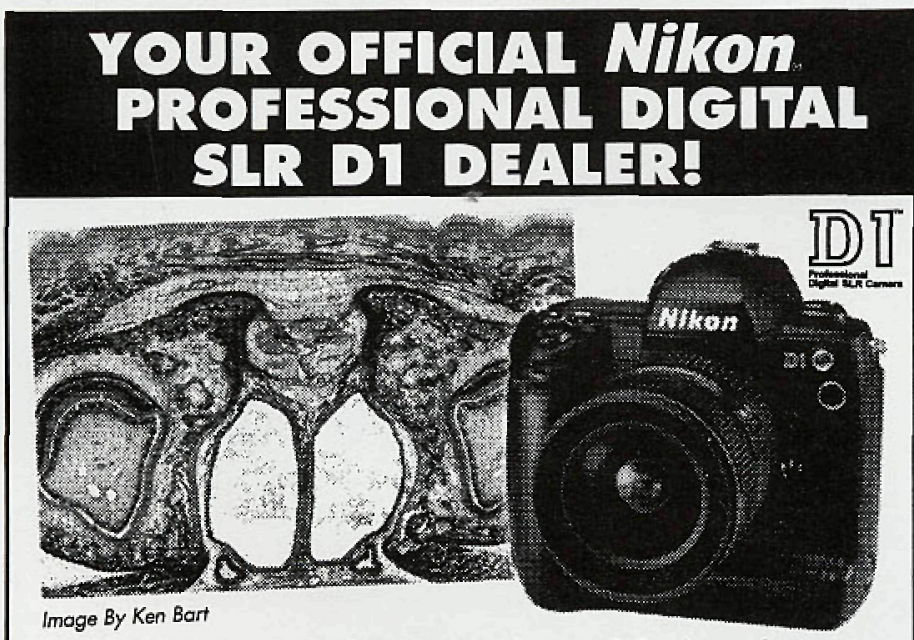

2.74-megapixel - Superhigh-speed, high-quality

4.5 frames per second - 3D Digital Matrix Image Control

High-speed AF system including Dynamic AF operation 3D Multi-Sensor Balanced Fill-Flash for D1

Compatible with virtually any F-mount Nikkor lens

$\$ 4,999$ Nikon DI Digital SLR Camera Set: DI Camera Bocty, EN-4 Battery Pack

MH-16 Quick Charger, Video Cable, Nikon View DX Browser, Nikon Video Cable for DI

DI Neck Strap, Instruction Manual, Nikon Limited One Year Warronty Document. "LENS SOID SEPARATELY

\section{Nikon. we take the words} Yours.
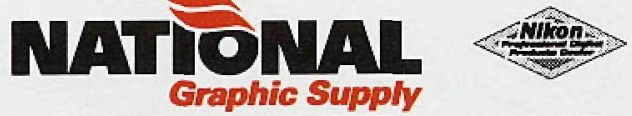

1.800 .223 .7130 ext.3109

226 North Allen Sireet, Albany, New York 12206 - Fax 800-832-2205 www.ngscorp.com - E-mail: scisales@ ngscorp.com binning should be adjusted so the desired (or achievable) resolution is 2 or 3 pixels. A typical configuration for mapping with 3d transition-metal $L$ edges used by the author is: $\alpha=2.9$ mrad, $\beta=4.8$ mrad, $i=200 \mathrm{nA}, \Delta=30 \mathrm{eV}, \mathrm{T}=15 \mathrm{~s}, 2 \mathrm{x}$ on-chip binning ( $512 \times 512$ pixels), $\sim 4000 x$ TEM magnification, $\sim 0.6 \mathrm{~nm} / \mathrm{pixel}, \delta \mathrm{d} \sim 1 \mathrm{~nm},>10^{4}$ CCD counts/pixel, and $\mathrm{t} / \lambda=0.5 \pm 0.2$ (where $\lambda$ is the inelastic scattering mean free path). Jump-ratio (2-window) and net core-loss intensity (3-window) maps are routinely produced and compared, in part to help detect artifacts. Zero-loss $I_{0}(\Delta<10 \mathrm{eV})$, low-loss $I_{k}$ (same $\Delta$ as used for core-loss images), and unfiltered images $I_{T}$ are also routinely recorded, as are electron energy-loss spectra (EELS). Local thickness is monitored from maps of $t / \lambda=\ln \left(I_{T} / I_{0}\right)$. Based on established EELS procedures, first-order correction of weak diffraction contrast can be made by dividing net core-loss intensity images ("elemental maps") by $I_{k}$, yielding areal concentrations. Further division by $t / \lambda$ (as an approximation for $t$ ) yields image intensities proportional to concentration in atoms $/ \mathrm{vol}^{2,5}$ This method assumes $\lambda$ is constant, often a good approximation for segregation in single-phase materials. However, defocusing the illumination to record $I_{0}, I_{k}$ and $I_{T}$ causes subtle changes in diffraction contrast and in the angular distribution of scattering, so the corrections are not exact. Alternatively, a ratio of net core-loss intensity maps for two elements minimizes diffraction contrast effects. For analytical situations where elemental concentration ratios are useful, this latter method is often more effective than the low-loss normalization scheme. ${ }^{16}$ Quantitative compositions are obtained with calculated ionization cross-sections or k-factors. Given the small window widths (typically $30 \mathrm{eV}$ ), which are contrary to good EELS practice, and the effects of near-edge structure (such as white lines) on the accuracy of calculated cross-sections, compositions are often surprisingly accurate.

An important performance parameter in elemental mapping is sensitivity. However, as in EELS, sensitivity depends critically on the particular combination of elements and edges; it is difficult to provide even general rules-of-thumb. The critical parameter determining sensitivity is the S/B ratio. The expected signal for a particular composition and set of measurement conditions is relatively well understood and can be calculated. The background is not as amenable to calculation since it arises from the tails of all the lower-energy edges, plasmons, and valence excitations. (Researchers are still waiting for the development of an "EELS simulator" that would be equivalent to DTSA for X-ray microanalysis.) The $L_{23}$ edges of the $3 d$ transition metals are well suited to EFTEM elemental mapping; they have large cross-sections, sharp onsets (some with strong white lines) and the background is generally well described by an inverse power law $\left(A E^{-r}\right)$. These desirable characteristics have been exploited to achieve high sensitivity measurements in steels, ${ }^{1,4,5}$ superalloys, ${ }^{2,3,5-8}$ and magnetic recording media. ${ }^{12,13,16}$ With their sharp onsets, $K$ edges are also well suited to EFTEM core-loss mapping, but their smaller cross sections can lead to poor sensitivity. For example, carbon and boron maps of precipitates in structural alloys are often of limited quality as a result of low S/B. ${ }^{5,7}$ Some $\mathrm{M}$ edges, especially those with white lines, provide high sensitivity. ${ }^{4,6}$ For edges with delayed maxima, however, background extrapolation becomes the limiting factor, especially in non-power-law regions such as $<200 \mathrm{eV}$, and reliability is degraded. Satisfactory jump-ratio maps can usually be produced but provide only qualitative information. ${ }^{4}$ For many elements the low-energy edges are unsuitable for 3-window mapping 


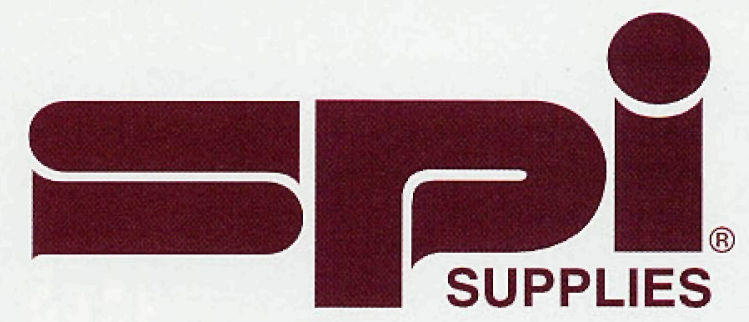

6 File Edit Diew Go Bookmarks Options Directory Help

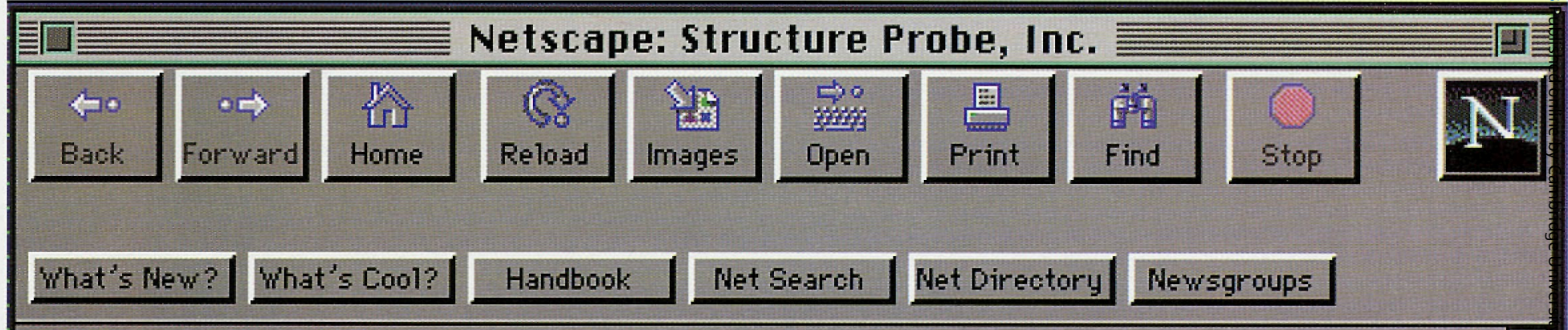
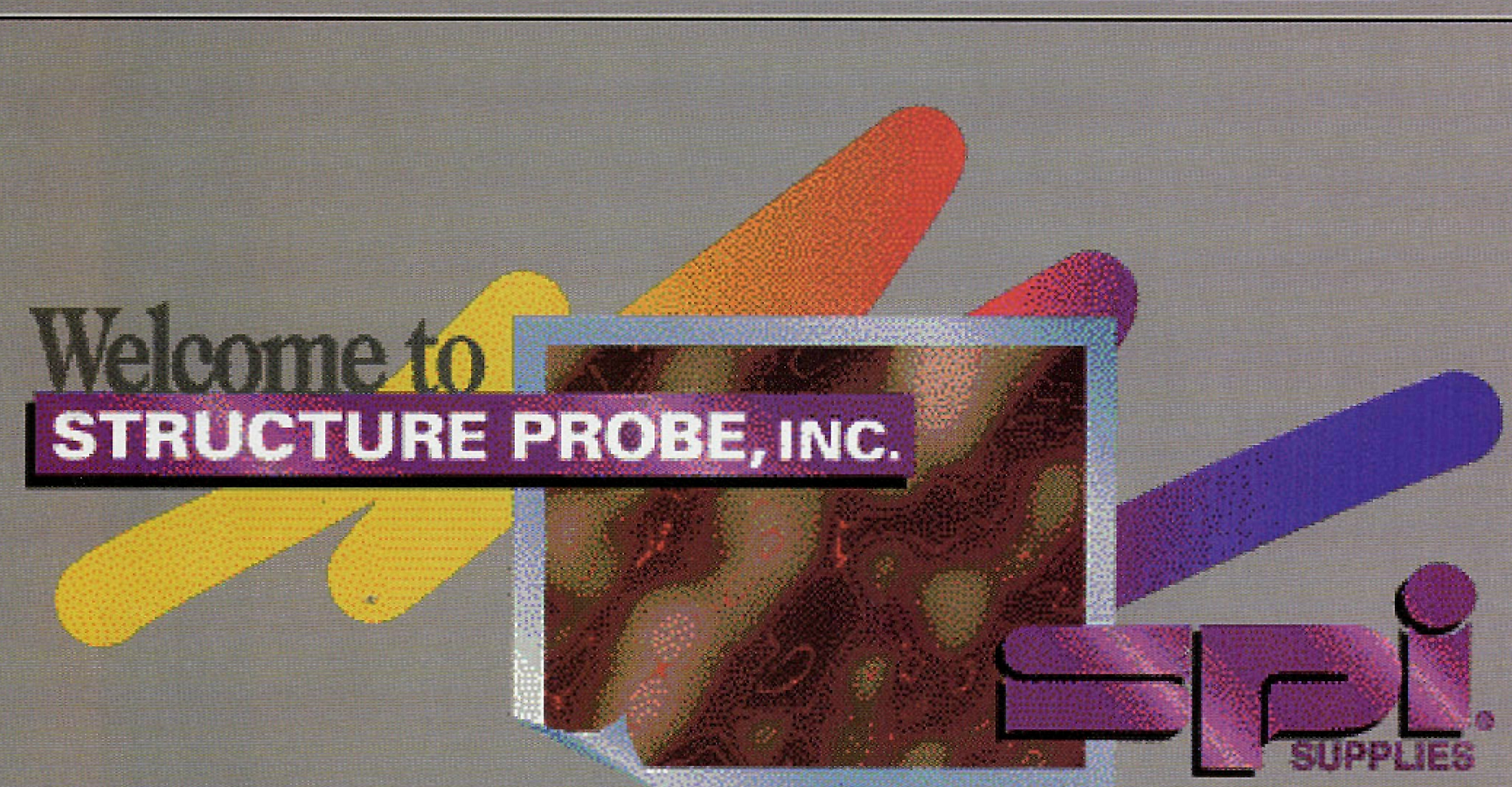

Discover The SourceBook Online

http://www.2spi.com

Up-To-The-Minute Information

For All Your Microscopy Needs

SPI Supplies Division of STRUCTURE PROBE, Inc.

P.O. Box 656 - West Chester, PA 19381-0656 USA

Ph.: 1-610-436-5400 • 1-800-2424-SPI (U.S. only) • FAX: 1-610-436-5755 • E-mail: spi2spi@ 2spi.com 
$\Delta$ Wide range of high performance detecting units

$\Delta$ Diverse number of integrated system offerings

$\triangle$ Comprehensive range of upgrade paths

\section{$\triangle$ EDAX is ISO 9001 certified}

\section{Over 30 years of $X$-ray experience}

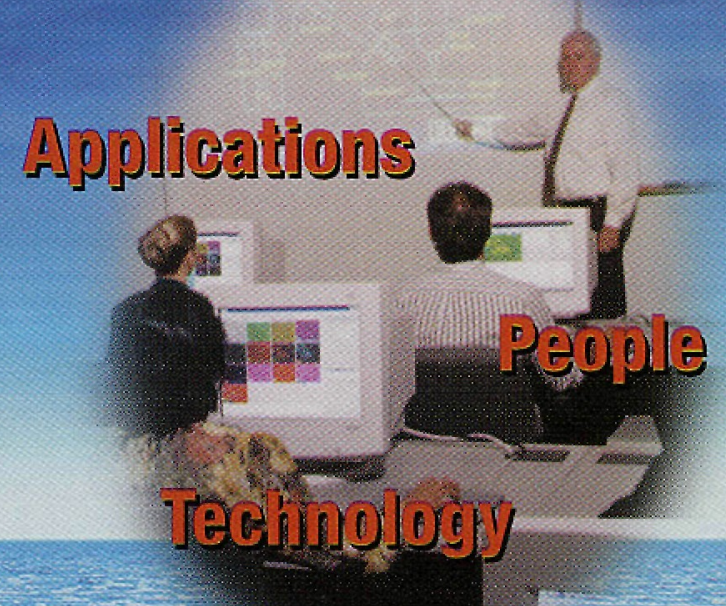

Visit our Web sitel http://www.edax.com

EDAX USA

91 McKee Drive

Mahwah, NJ 07430

Tel: 201.529.4880

Fax: 201.529.3156
EDAX Europe

Ringbaan Noord 103, P.0. Box 4144

5004 JC Tilburg, The Netherlands

Tel: +31.13 .536 .4000$

Fax: +31.13.535.6279
EDAX Japan

13-37, Kohnan 2-chome

Minato-ku, Tokyo 108 , Japan

Tel: +81.3 .3740 .5172$

Fax: +81.3 .3740 .5307$
EDAX South East Asia

Unit 55, 5/F, Hitec, 1 Trademart Drive

Kowloon Bay, Hong Kong

Tel: +852 2620-1127

Fax: +852 2620-1091

\section{ENERGY-FILTERED IMAGING}

Continued from page 22

and higher-energy edges produce too low a signal. Such elements are thus not amenable to high-resolution quantitative composition mapping with good sensitivity.

The long exposures and intense illumination commonly used for composition mapping mean specimen drift is almost unavoidable. Accurate alignment of the component images in a series is critical to avoid artifacts. Misalignments of just one pixel can lead to strong black-white (B-W) contrast. Even subpixel misalignment can result in $\mathrm{B}-\mathrm{W}$ artifacts at strong contrast gradients. As an alternative to cross-correlation, which often does not cope well with the contrast reversals in core-loss imaging, minimizing $\mathrm{B}-\mathrm{W}$ contrast effects (or quantitatively the variance) of the ratio of two images has been found to work reliably and sensitively, even for low-contrast fine image detail. Drift within the duration of each component exposure of a series has been reduced by the use of multiple shorter exposures at each energy-loss. Since the images in each sub-series should have identical contrast, alignment by (automated) cross-correlation is appropriate. A log-polynomial backgroundfitting scheme has been developed for use where power-law dependence is not sufficiently accurate, such as for energy losses $<200 \mathrm{eV} \cdot{ }^{14}$ Closely spaced edges present a challenge for EFTEM elemental mapping. For the case of $\mathrm{Cr}_{-} \mathrm{L}_{23}$ and $\mathrm{O}-\mathrm{K}$ edges, a 4-window method has been developed and applied with considerable success. Reliable $\mathrm{Cr}-\mathrm{L}_{23}$ intensities have been mapped for $\mathrm{Co}-\mathrm{Cr}$ based magnetic recording media ${ }^{16}$ and for ferrite in a dual-phase steel, ${ }^{17}$ both instances where surface oxide films on TEM specimens seem almost unavoidable. To achieve the optimum performance, deconvolution of the point spread function of the detector should be performed. This is especially true for sharp local minima in concentration where the "tails" from the adjacent high-concentration area(s) can lead to substantial increases in the apparent minimum concentration.

Near-edge fine structure can be a rich source of chemical bonding information. With narrow energy-selecting slits, it has been demonstrated that in favorable cases bonding information can be mapped with high spatial resolution by EFTEM. The valence of 3d transition metal cations in oxides has been mapped through the $L_{3} / L_{2}$ white line intensity ratio, which depends sensitively on valence. ${ }^{18,19}$ Differences in the ratio of $\mathrm{sp}^{2}$ to $\mathrm{sp}^{3}$ bonding in carbon films have been mapped through the ratio of $\pi^{*}$ to $\sigma^{*}$ intensities ${ }^{20}$ Such EFTEM methods have some advantages over methods based on scanning transmission electron microscopy (STEM). In fact, spectrum imaging by EFTEM rather than STEM should be seriously considered as the method of choice in situations where electron dose is not an issue and where data for many (e.g., $\left.10^{6}\right)$ image pixels are required.

Often in materials science, one-dimensional variations in composition or bonding normal to interfaces control material properties. Spectrum lines produced in the TEM mode with a GIF provide one-dimensional information on variations in fine structure (lowloss region or core-loss edges). ${ }^{9-11,20}$ This TEM spectrum-line method is based on the fact that in spectroscopy mode the GIF optics produce a line focus with one-dimensional spatial information retained normal to the energy dispersion direction. A $2 \times 0.5$ $\mathrm{mm} \mathrm{Au}$ slotted washer mounted in the GIF entrance aperture normal to the energy dispersion direction has been used to select image areas. Interfaces are aligned edge-on and normal to the slot 


\section{HIIGH BESOLUTION EDS DEIEGTOHS FOR TEM}

EDAX OFFERS COMPLETE SVSTEMU INTECHALIOW

EDAK FEATURES:

PCI Bus (32 bit) Architecture

Windows 98 or Windows NT

Digital Processing Standard

Superior Service \& Support

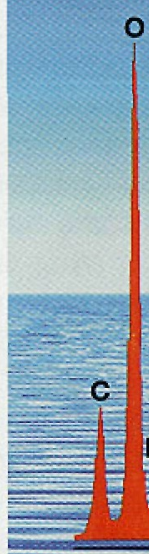

SUPERIOR PERFORMANCE.. GUARANTEED

$\triangle$ EDAM III electronics integrated with EMiSPEC at DLL. level

$\triangle$ Best standard resolution:

$132 \mathrm{eV}$ for $10 \mathrm{~mm}^{2}$

$136 \mathrm{eV}$ for $30 \mathrm{~mm}^{2}$

Resolution of $127 \mathrm{eV}$ and $132 \mathrm{eV}$ is typically achieved for $10 \mathrm{~mm}^{2}$ and $30 \mathrm{~mm}^{2}$ detecting units respectively!

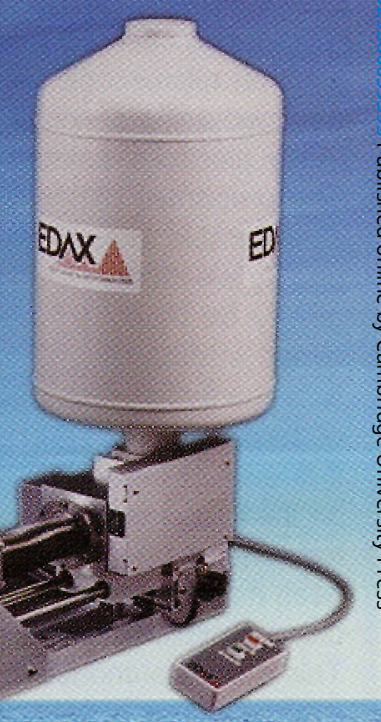

r-TEM Detector with a tilt-rotation specimen holder. The chief limitation of the TEM spectrum line method is that, just as in compositional mapping, spatial resolution is determined by chromatic aberration $\left[\delta=\mathrm{C}_{c} .(\Delta /\right.$ $E) . \beta$, where $E$ is the incident energy]. Thus, widely separated edges are incompatible with high spatial resolution, and $<1 \mathrm{~nm}$ is achievable only for a limited energy range (typically $20 \mathrm{eV}$ ).

Finally, in some applications, plasmon imaging can be used to monitor composition indirectly. The large signal means high sensitivity can be achieved and video-rate recording is possible. Again $\sim 1 \mathrm{~nm}$ resolution has been achieved, e.g., in studies of metallic Al particles in ion-implanted $\mathrm{MgAl}_{2} \mathrm{O}_{4}$ spinel. ${ }^{5}$ Background subtraction was even attempted with some success. For situations that produce only small plasmon shifts, effective EFTEM mapping may be precluded, but spectrum-lines at interfaces may offer advantages over the alternative, spectrum imaging in a STEM. ${ }^{21}$

1. J. Bentley and E.A. Kenik, Proc. Annual MSA Meeting $\mathbf{5 2}$ (1994) 1000.

2. J. Bentley et al., Proc. Microscopy and Microanalysis 1995, 268.

3. E.L. Hall and J. Bentley, Proc. Microscopy and Microanalysis $1995,260$.

4. J. Bentley et al., Proc. Microscopy and Microanalysis 1996 , 542.

5. J. Bentley et al., Proc. EMAG95, Inst. Phys. Conf. Ser. 147, Bristol, IOP (1995) 187.

6. J. Bentley, Microsc. Microanal. 3 (Suppl 2: Proceedings) (1997) 533.

7. J. Bentley et al., Microsc. Microanal. 3 (Suppl 2: Proceedings) (1997) 975.

8. E.L. Hall and J. Bentley, Mat. Res. Soc. Symp. Proc. 458
(1997) 121

9. J. Bentley and I.M. Anderson, Proc. Microscopy and Microanalysis $1996,532$.

10. I.M. Anderson and J. Bentley, Mat. Res. Soc. Symp. Proc. 458 (1997) 81.

11. I.M. Anderson and J. Bentley, Microsc. Microanal. 3 (Suppl 2: Proceedings) (1997) 931.

12. J. Bentley et al., Mat. Res. Soc. Symp. Proc. 517 (1998) 205.

13. J.E. Wittig et al., Mat. Res. Soc. Symp. Proc. 517 (1998) 211.

14. N.D. Evans and J. Bentley, Proc. Microscopy and Microanalysis 1996, 544 .

15. J. Bentley, Microsc. Microanal. 4 (Suppl 2: Proceedings) (1998) 158.

16. J. Bentley et al., Microsc. Microanal. 5 (Suppl 2: Proceedings) (1999) 136; ibid 634.

17. J. Bentley, Microsc. Microanal. 6 (Suppl 2: Proceedings) (2000) 362.

18. Z.L. Wang, J. Bentley and N.D. Evans, J. Phys. Chem. B 103 (1999) 751 .

19. Z.L. Wang, J. Bentley and N.D. Evans, Microsc. Microanal. 5 (Suppl 2: Proceedings) (1999) 102.

20. J. Bentley, K.C. Walter and N.D. Evans, Microsc. Microanal. 6 (Suppl 2: Proceedings) (2000) 154.

21. Research at the Oak Ridge National Laboratory (ORNL) SHaRE User Facility sponsored by the Division of Materials Sciences and Engineering, U.S. Department of Energy, under contract DE-AC05-00OR22725 with UT-Batelle LLC. Thanks to my many collaborators but especially to Drs. Ian Anderson, Ray Carpenter, Neal Evans, Ernie Hall, Ed Kenik, Matt Libera, Bruce Pint, Phil Rice, Renu Sharma, Z.L. Wang and Jim Wittig. 